\title{
Abordaje toraco-abdominal de la fístula broncobiliar
}

\author{
Ciro Andrés Murcia ${ }^{1}$, Jairo Eduardo Rivera², Gilberto Andrés Mejía², Carlos Andrés Benavides², \\ Juan Carlos Garzón ${ }^{3}$, Natalia Urueña ${ }^{1}$
}

Palabras clave: fístula biliar; fístula bronquial; conductos biliares; conducto colédoco; colangiocarcinoma.

\section{Resumen}

La fistula broncobiliar es una entidad poco usual en nuestro medio y poco descrita en la literatura cientifica. Se caracteriza por una comunicación anómala entre el árbol bronquial y la vía biliar, y se asocia a la presencia de bilis en el esputo (bilioptisis). Según su etiología, puede ser secundaria a la obstrucción distal de la vía biliar por patología benigna, maligna, infecciosa o iatrogénica.

Se presenta el caso de un paciente de 33 años, que cursó con una fístula broncobiliar secundaria a la derivación de la vía biliar percutánea por una lesión estenótica del confluente biliar, en quien -por medio de un abordaje torácico y abdominal-se practicó una lobectomía inferior por videotoracoscopia (Video-Assisted Thoracoscopy

Médico, residente de IV año de Cirugía General, Universidad de La Sabana, Chía, Colombia

2 Médico, cirujano hepatobiliar y de trasplantes, Unidad de Trasplantes, Fundación Cardioinfantil-Instituto de Cardiología, Bogotá, D.C., Colombia

3 Médico, cirujano de tórax, Fundación Cardioinfantil-Instituto de Cardiología, Bogotá, D.C., Colombia

Fecha de recibido: 22 de julio de 2016

Fecha de aprobación: 18 de noviembre de 2016

Citar como: Murcia CA, Rivera JE, Mejía GA, Benavides CA, Garzón JC, Urueña N. Abordaje toraco-abdominal de la fístula biliobronquial. Rev Colomb Cir. 2017;32:223-8.
Surgery, VATS) y hepatectomía con reconstrucción de la vía biliar por laparotomía.

Teniendo en cuenta lo inusual del caso, con pocos reportes en la literatura, se concluye la dificultad para establecer la posible etiología de las estenosis de la vía biliar en un paciente joven, sin poderse descartar la enfermedad maligna. Al hacer el diagnóstico, se debe tratar y corregir la causa; la cirugía sigue siendo el pilar del tratamiento y, con el advenimiento de la cirugía mínimamente invasiva, se puede hacer un manejo combinado para aprovechar los beneficios de la técnica, como se hizo en este caso.

\section{Introducción}

La fístula broncobiliar es una entidad poco común en nuestro medio. Descrita por primera vez en 1850 por Peacock ${ }^{1,2}$, se trata de una comunicación anómala entre la vía biliar y el árbol bronquial, y su sintomatología consiste en la presencia de bilis en el esputo (bilioptisis) y tos crónica. Puede presentarse como neumonía recurrente o con procesos infecciosos crónicos, y generar una morbilidad significativa para el paciente $(12,5 \%)^{3-5}$.

La persistencia de esta comunicación es favorecida por el gradiente de presión entre la presión positiva que se genera en la vía biliar y la presión negativa del espacio torácico. La bilis que se fuga del árbol biliar actúa como agente irritante, causa inflamación y genera un proceso crónico y necrosis del diafragma ${ }^{5}$. Entre las posibles causas, se encuentran: infección local, condición 
congénita, trauma, obstrucción o neoplasia de la vía biliar, trauma hepático, absceso subfrénico y carcinoma hepatocelular ${ }^{3-5}$.

Crnjac, et al., proponen agrupar las posibles causas en cinco grupos: 1) fístula broncobiliar o pleurobiliar congénita; 2) enfermedad hepática hidatídica o abscesos (equinocócica, amebiana o piógena); 3) obstrucciones de la vía biliar (iatrogénica); 4) lesiones traumáticas (iatrogénica, contundente o penetrante), y 5) fístulas iatrogénicas (resecciones hepáticas, ablación por radiofrecuencia, irradiación y drenajes torácicos) 3,4.

\section{Reporte de caso}

Se trata de un paciente de sexo masculino de 33 años de edad, con un síndrome biliar obstructivo por estenosis del confluente hepático, con sospecha de neoplasia, la cual no pudo confirmarse mediante biopsia; se requirió un procedimiento endoscópico derivativo y derivación externa por vía percutánea.

Al retirar la derivación, presentó bilioptisis durante ocho meses, motivo por el cual fue valorado por nuestro servicio. En el momento de la valoración, refirió pérdida de $11 \mathrm{~kg}$ de peso y el índice de masa corporal (IMC) era entonces de $17,1 \mathrm{~kg} / \mathrm{m}^{2}$. Presentaba, además, coluria, acolia e ictericia generalizada.

En los exámenes paraclínicos de control se encontró: albúmina, $2,5 \mathrm{~g} / \mathrm{dl}$; fosfatasa alcalina, $1.094 \mathrm{U} / \mathrm{L}$; bilirrubina total, $5,5 \mathrm{mg} / \mathrm{dl}$ y directa de $4,3 \mathrm{mg} / \mathrm{dl}$, y los marcadores tumorales (alfa-fetoproteína, Ca19,9 y antígeno carcinoembrionario) fueron negativos. En la tomografía computarizada (TC) de tórax se observó un extenso compromiso parenquimatoso pulmonar en el lóbulo inferior derecho, tabiques en la pleura parietal hacia el aspecto medial del lóbulo inferior derecho y una zona que podría corresponder a una neumonía necrosante (figura 1). Se hospitalizó para estudio y tratamiento.

En la colangiopancreatografía endoscópica retrógrada (CPRE) se encontraron múltiples irregularidades y dilataciones saculares por colangitis, y una colección en los segmentos superiores del hígado e importante disminución del calibre del conducto hepático común (figura 2).

En la resonancia magnética (RM) de abdomen, con contraste (Primovist ${ }^{\circledR}$, gadoxetato disódico, Bayer), se evidenciaron múltiples abscesos, uno de ellos con compromiso extrahepático subdiafragmático y en íntima relación con el lóbulo pulmonar inferior derecho. Además, se observó obstrucción segmentaria del árbol biliar, derecho e izquierdo, con engrosamiento concéntrico de las paredes, realce tardío y sin evidencia de masa, cambios que sugerían un proceso inflamatorio antes que una lesión neoplásica (figura 3).

Se sometió a cirugía después de mejorar su estado nutricional, y se encontró una fístula broncobiliar de 1 cm de diámetro, con hepatización del lóbulo pulmonar inferior derecho, sin identificar ningún compromiso metastásico; además, se encontraron cambios atróficos y cicatriciales en el lóbulo hepático izquierdo, asociados con el proceso previo de colangitis. No se evidenció compromiso por enfermedad maligna extrahepática.

$\mathrm{El}$ abordaje fue toracoscópico y asistido por video ( $\mathrm{Vi}$ deo-Assisted Thoracoscopy Surgery, VATS) y se practicaron lobectomía pulmonar inferior derecha y "decorticación" (o pleurectomía) (figura 4a). El abdomen se abordó por laparotomía y se decidió asociar una lobectomía hepática

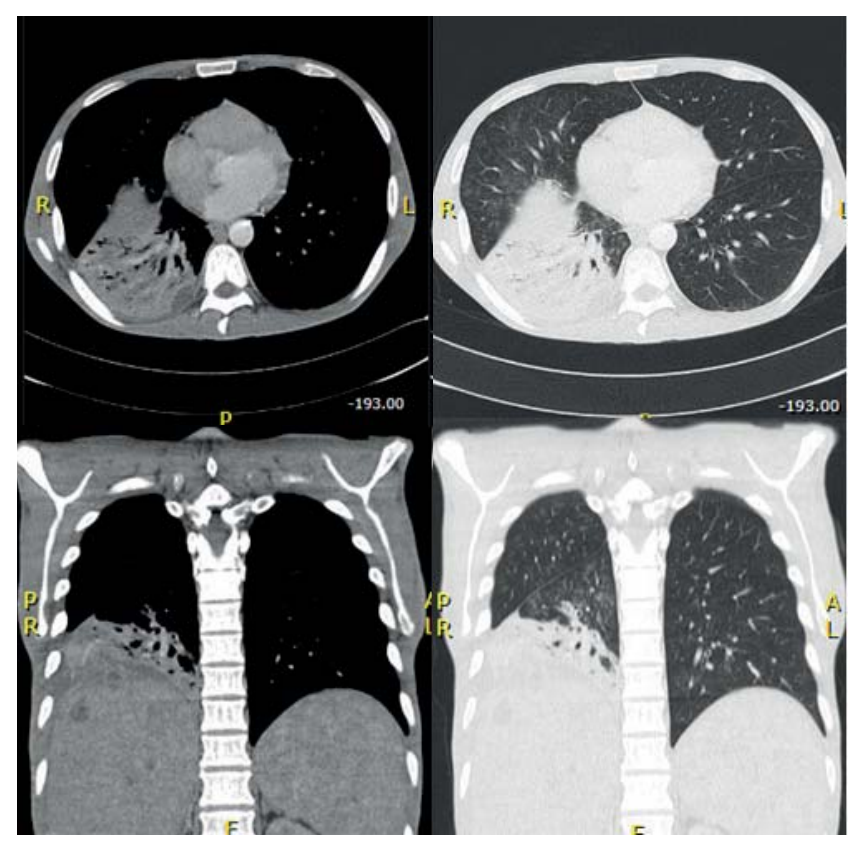

FIgURA 1. Tomografía computarizada de tórax: cortes axiales mediante técnica helicoidal y reconstrucciones de $3 \mathrm{~mm}$, después de la administración del medio de contraste intravenoso $(75 \mathrm{ml}$ de iohexol) extenso compromiso parenquimatoso pulmonar en el lóbulo inferior derecho, tabiques en la pleura parietal hacia el aspecto medial del lóbulo inferior derecho y una zona que podría corresponder a una neumonía necrosante. 


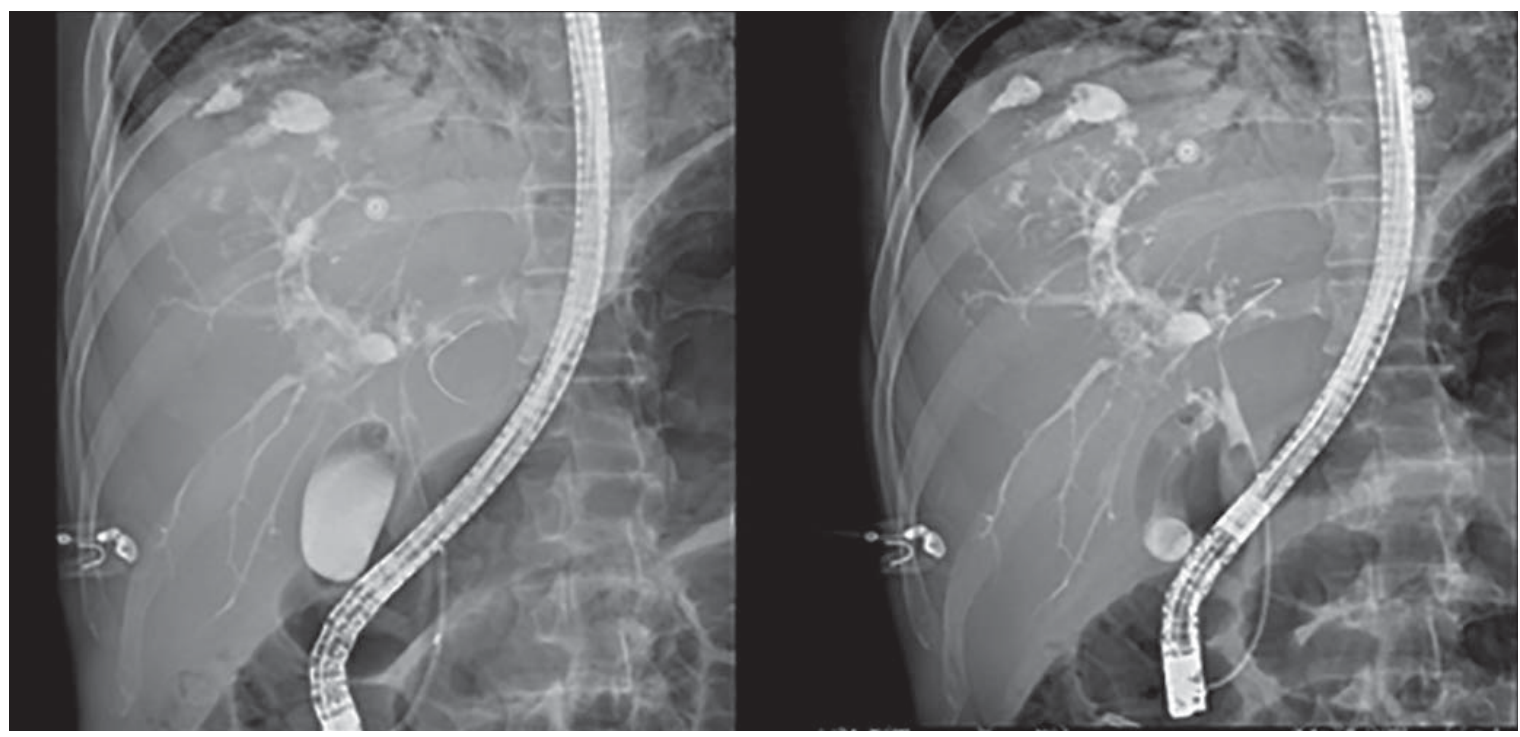

FigURA 2. CPRE: múltiples irregularidades y dilataciones saculares por colangitis, con formación de una colección en los segmentos superiores, y disminución importante del calibre del conducto hepático común.
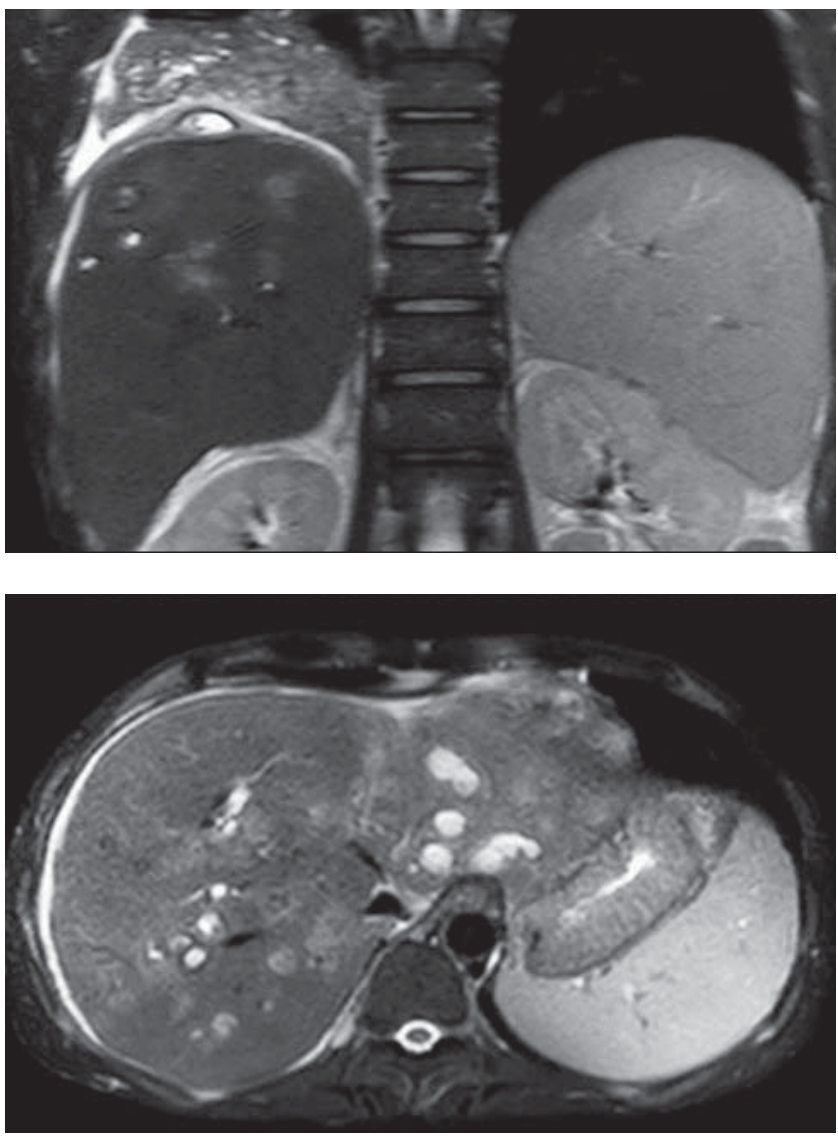

Figura 3. Resonancia magnética de abdomen con Primovist ${ }^{\circledR}$ donde se evidencian múltiples abscesos, uno de ellos con compromiso extrahepático subdiafragmático y en íntima relación con el lóbulo pulmonar inferior derecho.
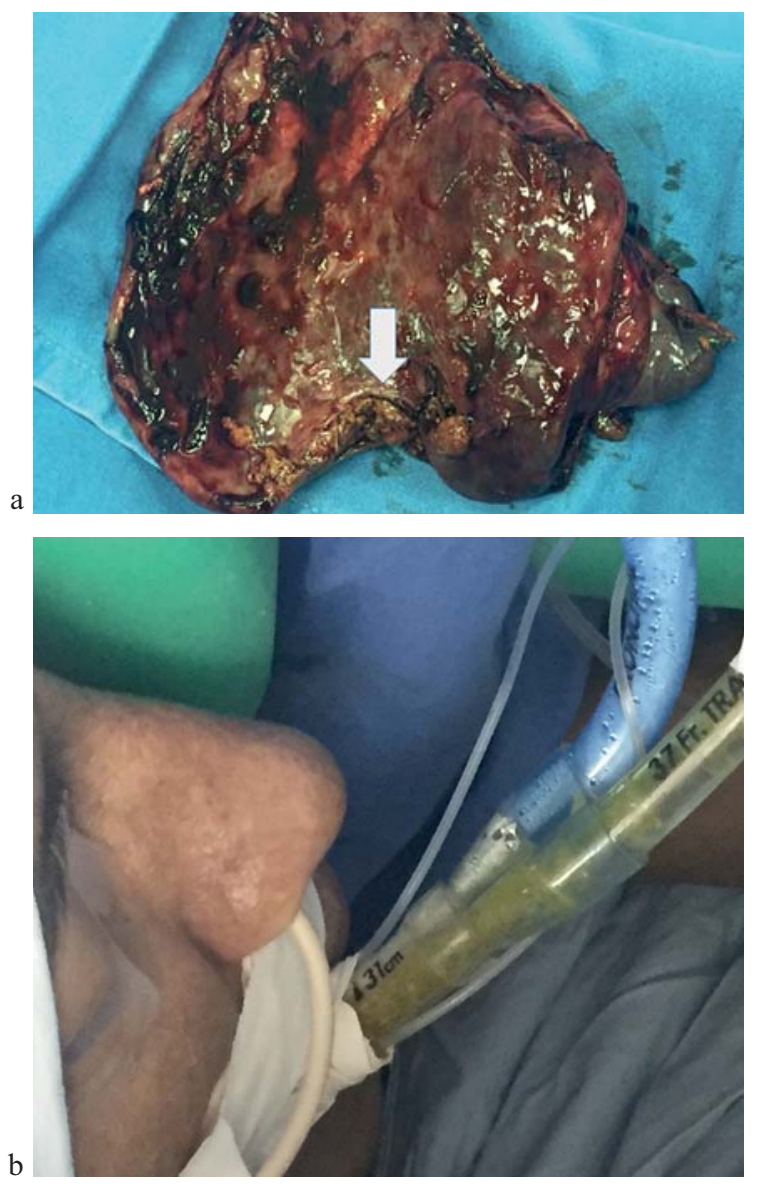

Figura 4. a. Pieza quirúrgica de la lobectomía inferior derecha: fistula en su borde inferior (flecha). b. Presencia de bilis en el tubo de doble luz. 
izquierda y una resección de la vía biliar extrahepática, con reconstrucción mediante hepato-yeyunostomía. En el postoperatorio inmediato, presentó una fuga de bilis que se resolvió de manera espontánea.

La histopatología reportó un tumor de tipo colangiocarcinoma, de 2,5 x 1,5 cm, con un patrón de crecimiento mixto y formación de masa e infiltración periductal que invadía la superficie peritoneal visceral, con márgenes de la sección del parénquima comprometido y presencia de invasión perineural. En la pieza quirúrgica pulmonar se evidenció bronconeumonía con presencia de bilis dentro de los alvéolos.

La hospitalización fue de 33 días. El paciente se controló ambulatoriamente por el Servicio de Oncología en su ciudad de origen, y no ha habido evidencia de progresión de la enfermedad en los 10 meses después de la cirugía.

\section{Discusión}

La fístula broncobiliar se define como una comunicación anómala entre la vía biliar y el árbol bronquial, sin importar su etiología ${ }^{5}$ (figura 5). Fue descrita por primera vez en 1850 por Peacock, como la complicación de un quiste hidatidiforme del hígado ${ }^{1,2}$. En 1938, Oschsner describió una incidencia de $4 \%$ de fístula broncobiliar en 453 abscesos hepáticos piógenos y de $10,5 \%$ en 3.608 abscesos subfrénicos ${ }^{4}$.

En la revisión bibliográfica, se encontraron múltiples reportes de Ferguson y Burford sobre casos, desde 1967 hasta años recientes, en los cuales se expusieron el enfoque, el tratamiento, la etiología y la evolución ${ }^{2,6,7}$. La etiología de esta entidad está descrita principalmente en los procesos infecciosos (hidatídico, equinocócico, amebiano). Según Morton y Phillips, en segundo lugar se encuentra la enfermedad de la vía biliar, usualmente obstructiva $^{5}$, la cual se perpetúa por la diferencia entre los gradientes de presión que se generan en la vía biliar y el espacio bronco-pleural, además de la presión generada por el esfínter de Oddi ${ }^{8-10}$, el cual funciona como una válvula unidireccional. Otras causas que se deben tener en cuenta al momento del estudio de un paciente con sospecha de esta entidad, es el antecedente de trauma toraco-abdominal, la tuberculosis, la sífilis, la enfermedad de Hodgkin, la ascaridiasis y, con menor frecuencia, las fístulas de origen congénito ${ }^{3,5}$.
El diagnóstico es predominantemente clínico y está dado por la presencia de bilis en el esputo, conocida como bilioptisis (figura 4b) ${ }^{11}$, condición que genera neumonitis aguda o tos crónica irritativa. Cabe anotar que, en algunas enfermedades, se pueden presentar síntomas similares, pero con menor gravedad. Sutherland describió la bilioptisis en un paciente con enfermedad de células falciformes y crisis hemolíticas; por esto, una vez se hayan descartado dichas entidades o se tenga claro el antecedente de lesión de la vía biliar, el diagnóstico se orienta específicamente a una fístula ${ }^{4-7}$.

El tratamiento actual se divide en tres categorías: quirúrgico, conservador y combinado. El principal pilar del tratamiento sustentado en la literatura científica es la cirugía, aunque se debe determinar la etiología de la fístula y el estado del paciente, con el fin de tomar la mejor decisión para resolver del problema ${ }^{12-15}$.

Generalmente, la intervención quirúrgica incluye resección pulmonar -desde una cuña, hasta lobectomía del segmento comprometido-, resección del trayecto fistuloso, seguido de cierre del defecto diafragmático, y corrección de la causa en el hígado ${ }^{6,15-17}$. Se han descrito diversos abordajes quirúrgicos, como toracofrenolaparotomía (una sola incisión para abordaje de las dos cavidades), abordaje separado (toracotomía y laparotomía) y, con el advenimiento de la cirugía mínimamente invasiva, cirugía toracoscópica con asistencia de video (VATS) y laparotomía ${ }^{6}$.

El manejo conservador incluye la CPRE, con drenaje nasobiliar o sin él, la generación de embolia de la fístula con Histoacryl ${ }^{\circledR}$ o el drenaje percutáneo transparietohepático, así como el uso de ocreótido para disminuir la secreción de bilis; asimismo, tratamientos combinados que incluyen CPRE y drenaje percutáneo, y derivaciones por imagenología mientras se estabiliza y se recupera nutricionalmente el paciente para la cirugía ${ }^{6,12,15-17}$.

Se trata de una entidad de muy baja incidencia en la que el diagnóstico definitivo se obtuvo únicamente después de completar la resección de la lesión y la lectura de la histopatología.

No obstante haber practicado todos los estudios diagnósticos a nuestro alcance (imágenes y marcadores tumorales), y sin tener ningún indicio de que fuera una enfermedad maligna, se optó por la recuperación 
nutricional y el manejo antibiótico antes de la cirugía. A pesar del infortunado reporte de patología (resección R2), consideramos que la cirugía practicada fue la mejor opción para el paciente, pues se logró una paliación completa de su cuadro clínico y se presentó una evolución satisfactoria que favoreció el inicio temprano del tratamiento oncológico.

\section{Conclusión}

Aunque es una entidad poco común en nuestro medio, la fístula biliar genera una alta morbimortalidad, al igual que un deterioro importante de la calidad de vida, principalmente por la bilioptisis. Una vez hecho el diagnóstico y -si es posible- determinada la etiología, debe escogerse el mejor tratamiento posible para cada paciente. Los métodos menos invasivos, como la CPRE y la derivación biliar percutánea, tienen cabida como pasos previos a la corrección quirúrgica de esta entidad y, solo en una minoría de los casos, conllevan su resolución.

La cirugía sigue siendo el pilar del tratamiento y, con el advenimiento de la cirugía mínimamente invasiva, se puede hacer un tratamiento combinado para aprovechar los beneficios de esta técnica, como se hizo en el presente caso, en el cual se logró mejorar la calidad de vida del paciente, con la resolución completa de la bilioptisis y del cuadro biliar obstructivo, lo que le ha permitido permanecer con un esquema de quimioterapia paliativa de mantenimiento.

\title{
Thoraco-abdominal approach in bronchobiliary fistula
}

\begin{abstract}
Bronchobiliary fistula is a rare entity in our environment and it is poorly described in the scientific literature. It is characterized by an abnormal communication between the bronchial tree and the biliary tract, and is associated with the presence of bile in the sputum (bilioptysis). According to its etiology, it may be secondary to a distal obstruction of the bile duct due to a benign, malignant, infectious, or iatrogenic pathology. We present the case of a 33-year-old patient who had a bronchobiliary fistula secondary to a percutaneous biliary tract bypass due to a stenotic lesion of the biliary confluent in whom an inferior lobectomy by video-assisted thoracoscopy surgery (VATS) was performed with hepatectomy with biliary tract reconstruction by laparotomy, a combined thoracic and abdominal approach. Given the low incidence of this type of cases and the scarce literature reports, our conclusion is that it is difficult to define the possible etiology of the bile duct stenosis in young patients, and that a malignant pathology cannot be ruled out. Once the diagnosis is established, the cause should be treated. Surgery remains the mainstay of treatment, and with the advent of minimally invasive surgery a combined type of management can be performed so as to take advantage of the benefits of this technology.
\end{abstract}

Key words: Biliary fistula; bronchial fistula; bile ducts; common bile duct; cholangiocarcinoma.

\section{Referencias}

1. Saylam A, Ersoy U, Baris I, Artvinli M, Bozer AY. Thoracobiliary fistulas. Br J Dis Chest. 1974;68:264-72.

2. Ferguson TB, Burford TH. Pleurobiliary and bronchobiliary fistulas. Arch Surg. 1967;5:380-6.

3. Aguilar C, Cano R, Camasca A, Gonzales J, Rivera J, Del Pino $\mathrm{T}$, et al. Fístula broncobiliar congénita detectada por colescintigrafía. Revista de Gastroenterología del Perú. 2005;25:216-8.
4. Crnjac A, Pivec V, Ivanecz A. Thoracobiliary fistulas: Literature review and a case report of fistula closure with omentum majus. Radiol Oncol. 2013;47:77-85.

5. Gugenheim J, Ciardullo M, Traynor O, Bismuth H. Bronchobiliary fistulas in adults. Ann Surg. 1988;207:90-4.

6. Belaidi M, Wang YZ, Joshi V. A 60 year old man with chronic cough and green sputum. Gastroenterology. 2014;147:575-6. 
7. Singh B, Moodley J, Sheik-Gafoor MH, Dhooma N, Reddi A. Conservative management of thoracobiliary fistula. Ann Thorac Surg. 2002;73:1088-91.

8. Mukkada RJ, Antony R, Francis JV, Chettupuzha AP, Augustine $\mathrm{P}$, Venugopal B, et al. Bronchobiliary fistula treated successfully with endoscopic microcoils and glue. Ann Thorac Surg. 2014;98:e33-4.

9. Mitra S, Bhatia N, Dey N, Dalal U. Bronchobiliary fistula: An anesthetic challenge. J Clin Anesth. 2009;21:360-2.

10. Olivencia-Yurvati AH, Rollins C. Bilorrhea secondary to bronchobiliary fistula. Int Surg. 2014;99:438-41.

11. Vimalraj V, Jeswanth S, Selvakumar E, Jyotibasu D, Rajendran S, Ravichandran P. A case of recurrent biliptysis. J Thorac Cardiovasc Surg. 2007;133:1662-3.

12. Yilmaz U, Sahin B, Hilmioglu F, Tezel A, Boyacioglu S, Cumhur T. Endoscopic treatment of bronchobiliary fistula: Report on 11 cases. Hepatogastroenterology. 1996;43:293-300.

13. Beslic S, Zukic F, Milisic S. Percutaneous transthoracic CT guided biopsies of lung lesions; fine needle aspiration biopsy versus core biopsy, Radiol Oncol. 2012;46:19-22.
14. Burmeister S, Krige JE, Bornman PC, Nicol AJ, Navsaria P. Endoscopic treatment of persistent thoracobiliary fistulae after penetrating liver trauma. HPB (Oxford). 2009;11:171-5.

15. Navsaria $\mathrm{PH}$, Adams S, Nicol AJ. Traumatic thoracobiliary fistulae: A case report with a review of the current management options. Injury Int J Care Injured. 2002;33:639-43.

16. Sheik-Gafoor MH, Singh B, Moodley J. Traumatic thoracobiliary fistula: Report of a case successfully managed conservatively, with an overview of current diagnostic and therapeutic options. J Trauma. 1998;45:819-21.

17. Oparah SS, Mandal AK. Traumatic thoracobiliary (pleurobiliary and bronchobiliary) fistulas: Clinical and review study. J Trauma. 1978;18:539-44.

Correspondencia: Jairo Eduardo Rivera, MD jarivera@cardioinfantil.org Bogotá, D.C., Colombia 